\title{
A METHOD OF COMPLEX CALCULATION OF RATIONAL STRUCTURAL PARAMETERS OF RAILWAY HUMPS
}

\author{
Sergit PAnchenko ${ }^{a}$, Oleksandr Ohar $^{b}$, MAKsym Kutsenko $^{c}$, \\ JULIA SMACHILO ${ }^{c, *}$ \\ ${ }^{a}$ Rector, Ukrainian State University of Railway Transport, Feyerbach square 7, Kharkiv, Ukraine \\ ${ }^{b}$ Head of Department of Railway Station and Junctions, Ukrainian State University of Railway Transport, \\ Feyerbach square 7, Kharkiv, Ukraine \\ ${ }^{c}$ Department of Railway Station and Junctions, Ukrainian State University of Railway Transport, Feyerbach \\ square 7, Kharkiv, Ukraine \\ * corresponding author: smachilo.julia@gmail.com
}

\begin{abstract}
The article deals with a method of complex calculation of rational structural parameters of humps at classification yards. Unlike existing methods, this method allows an implementation of the technology of guided gravity regulation of the cut speed by applying a special layout and profile arrangement. The authors believe that it will decrease the maintenance costs to refund damaged car and cargo, costs on electricity needed for the cut speed regulation and some extra charges due to demurrages caused by waiting for breaking-up at arrival yards.
\end{abstract}

KEYWORDS: railway transport, railway humps, optimization.

\section{INTRODUCTION}

Due to certain trends in the world energy resources market and severe competition in transportation, the research of optimization of rail transportation charges is urgent. Besides, the problem is also of importance for sorting operations at railway stations, as their parameters are greatly conditioned by structural parameters of the humps. In article [1, the authors pay an attention to the fact that one of the components considerably influencing the total costs of transportation are the car processing costs on humps. Cars can be processed several times on their way from departure stations to destination stations. According to [1, a lot of factors influence the processing costs, among which are the costs of depreciation, spare parts and maintenance of cut speed regulators (the maintenance costs are proportional to the costs of the regulators).

Over a long period of time a lot of scientists have paid their attention to an improved layout and profile structure of a hump, cut rolling speed regulators, systems of automated hump technological processes and cut braking modes to increase the breaking-up efficiency [2 [7]. Thus, in research [2, 3], the aim is achieved by the optimized structures of hump necks, in [4] - by defining the optimal parameters of the longitudinal hump profile, in [5] - by developing new and improved existing structures for cut rolling speed regulators, in [6] by forming approaches to the automated braking regulation, and in [7] — by optimizing cut braking modes. The analysis of the above-mentioned sources testifies that many scientists theoretically prove the possibility to increase the breaking-up efficiency with the methods proposed by the authors for humps.

Research 8 implies that there are some factors of the sorting process substantially influencing the breaking-up efficiency; thought they are rather difficult to consider, forecast or formalize. The operational condition of cut rolling speed regulators and automation devices, the degree of consideration and presentation method of a random nature, the state of wheelsets and other factors are among them. The research concluded that the reduction of influence of a human factor on the breaking-up efficiency is still a problem. Thus, consideration, forecasting, or formalization of the above-mentioned factors is currently a very difficult challenge, and the solution is not found yet.

Also, in research [8], the authors substantiate the importance to implement the technology of guided gravity braking for cuts. The special layout and profile arrangement for classification yards proposed by the authors (Figure 1) can be used to implement the given technology.

A special feature of such an arrangement is the location of the switching area (SA) (either in part or in whole) at the origin of sorting tracks up to the yard retarder position (YRP) on the ascent. The other elements on the section from the hump crest (HC) to the design point (DP) were located on the descent. The height and longitudinal profile of the arrangement provide, firstly, the rolling of slow light car (SL) in unfavourable winter conditions from HC to DP, the most difficult track in terms of resistance, and, secondly, sufficient intervals at dividing switching points in the SL-FH link (where FH- fast heavy car). 


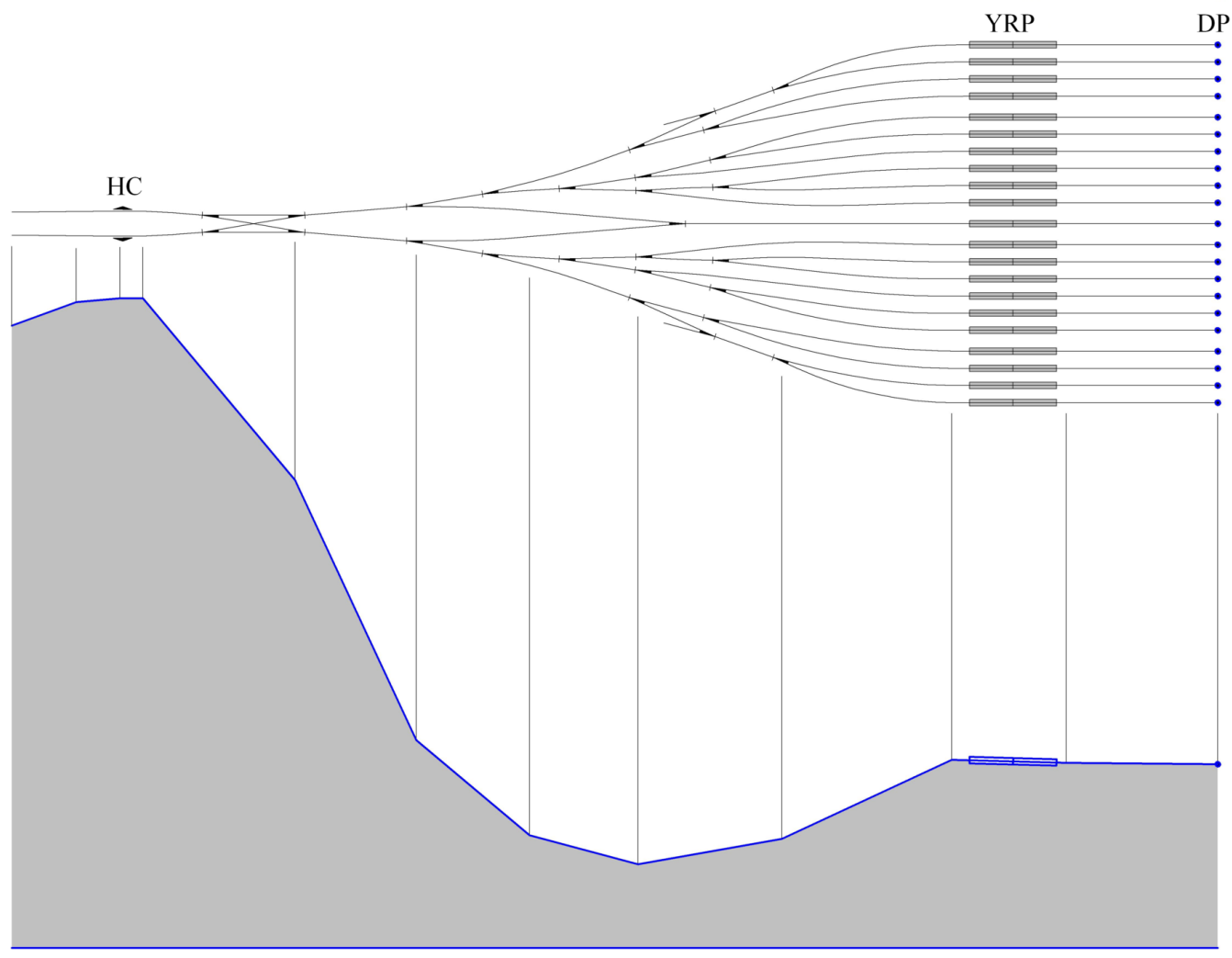

FIGURE 1. The arrangement of the guided gravity regulation of cut braking.

Therefore, intervals between cuts, sufficient for throwing over points from one position to another, are only provided due to a special structure of the descent part profile, and the location of certain profile elements on the ascent allows slowing the cars. In other words, such an arrangement generates the gravity braking effect. When applying this sorting arrangement, YRP does not change its functionality.

In [8], the authors put forward the hypothesis that under automated car processing, the accrued economic benefit over the calculation operational period for the arrangement will exceed the accrued economic benefit over the similar operational period for a conventional automated sorting hump. Even if the costs of cut rolling speed regulators can be twice as higher when applying the technology of guided gravity regulation of cut braking (additional investment into automated devices for conventional humps can compensate the difference of investment into car retarders).

\section{A METHOD OF COMPLEX CALCULATION OF RATIONAL STRUCTURAL PARAMETERS OF RAILWAY HUMPS}

Therefore, the most efficient structural variant for a hump design with the guided gravity regulation of cut braking is the variant of the least needed capacity of the YRP ( $\left.H_{\mathrm{YRP}}\right)$ in terms of meeting requirements for safety and fail-safe sorting operations. Thus, the needed capacity of the YRP is the criterion for rationalized structural parameters of a hump with the guided gravity braking technology for cuts. As far as for a certain hump, if the position of its crest and profile element length are constants, then:

$$
H_{\mathrm{YRP}}=f\left(I_{1}, I_{2}, \ldots, I_{n}\right)
$$

where $I_{1}, I_{2}, \ldots, I_{n}$ - the slopes of section elements from the hump crest to the origin of the switching area.

Let us develop the objective function to define the rational values of structural parameters of a hump.

Under favourable rolling conditions, the needed capacity of the yard retarder position, according to [1], is defined as follows:

$$
\begin{aligned}
& H_{\mathrm{YRP}}=k_{e n}\left(\left(\sum_{r=1}^{n} L_{r} I_{r}+L_{\mathrm{switch}} i_{\mathrm{switch}}+\triangle L_{\mathrm{st}} i_{\mathrm{st}}\right) \cdot 10^{-3}+\frac{V_{0}^{2}}{2 \cdot g^{\prime}}\right) \\
= & \left.\left(w_{0} L_{\mathrm{HC}-\mathrm{SA}}+V_{\mathrm{mid}(\mathrm{HC}-\mathrm{SA})}^{2} \cdot\left(0.56 \cdot n_{\mathrm{HC}-\mathrm{SA}}+0.23 \cdot \sum \alpha_{\mathrm{HC}-\mathrm{SA}}\right)\right) \cdot 10^{-3}-\left(L_{\mathrm{switch}} i_{\mathrm{switch}}+\triangle L_{\mathrm{st}} i_{\mathrm{st}}\right) \cdot 10^{-3}\right),
\end{aligned}
$$


where $k_{e n}$ - the enlargement factor of the minimal design capacity of retarder positions on the hump descent; $n$ - the number of profile elements of the descend (from the hump crest to the origin of the switching area); $L_{r}, I_{r}-$ the length, $m$ and the slope, $\%_{0}$ of the profile elements of a hump from the crest to the origin of the switching area), respectively; $L_{\mathrm{switch}}, I_{\mathrm{switch}}$ - the length, $m$ and the slope, $\%$ of the switching area, respectively; $\triangle L_{\mathrm{st}}, i_{\mathrm{st}}$ - the length, $m$ and the slope, $\%$ of the sorting track section from the switching area to the origin of YRP; $V_{0}-$ the initial speed of the consist shunting on the hump crest, $\mathrm{m} / \mathrm{s} ; \mathrm{g}^{\prime}$ - the acceleration of the gravity force of a heavy car with consideration of the rotating wheelset masses, $m / s^{2} ; w_{0}$ - the basic specific resistance to a heavy car, $N / k N ; L_{\mathrm{HC}-\mathrm{SA}}$ - the section length from the hump crest to the origin of the switching area, $m ; V_{\mathrm{mid}(\mathrm{HC}-\mathrm{SA})}^{2}$ - the average speed of a heavy car on the section from the hump crest to the origin of the switching area, $m / s$; $n_{\mathrm{HC}-\mathrm{SA}}$ - the number of switching points on the section from the hump crest to the origin to the switching area; $\sum \alpha_{\mathrm{HC}-\mathrm{SA}}-$ the sum of the rotation angles on the section from the hump crest to the origin of the switching area.

Since each profile element consists of technological elements:

$$
\begin{gathered}
\sum_{r=1}^{n} L_{r} I_{r}=\sum_{i=1}^{m} L_{i} i_{i}, \\
V_{\operatorname{mid}(\mathrm{HC}-\mathrm{SA})}^{2}\left(0.56 \cdot n_{\mathrm{HC}-\mathrm{SA}}+0.23 \cdot \sum \alpha_{\mathrm{HC}-\mathrm{SA}}\right)=\sum_{i=1}^{m}\left(0.56 \cdot n_{i}+0.23 \cdot \sum a_{i}\right) \cdot V_{\operatorname{mid}(i)}^{2},
\end{gathered}
$$

where $m$ - the number of technological elements.

The values $V_{0}, g^{\prime}, w_{0}, L_{i}, n_{i}, \sum \alpha_{i}$ for $i=1, \ldots, m$ are constants.

Let:

$$
k_{e n}=A, \quad \frac{V_{0}^{2}}{2 \cdot g^{\prime}}-L_{\mathrm{HC}-\mathrm{SA}} w_{0} \cdot 10^{-3}=B, \quad\left(0.56 \cdot n_{i}+0.23 \cdot \sum \alpha_{i}\right) \cdot 10^{-3}=C_{i},
$$

then

$$
H_{\mathrm{YRP}}=A\left(\sum_{i=1}^{m}\left(L_{i} i_{i}\right) \cdot 10^{-3}+B+\sum_{i=1}^{m} C_{i} \cdot V_{\operatorname{mid}(i)}^{2}\right) \rightarrow H_{\mathrm{YRP}(\min )} .
$$

The average rolling speed of a heavy car on $i$ th technological element is:

$$
V_{\operatorname{mid}(i)}=\frac{V_{i}^{\prime}+V_{i-1}}{2}
$$

where $V_{i}^{\prime}$ - the speed of a heavy car at the end of $i$ th element in a first approximation (calculation of $V_{i}^{\prime}$ considers only those specific resistances, which do not depend on the average rolling speed on a technological element: basic $\left(w_{0(i)}\right)$, on snow and frost $\left(w_{\mathrm{sn}(i)}\right)$ and on braking $\left.\left(w_{\mathrm{b}(i)}\right)\right), m / s$ :

$$
V_{i}^{\prime}=\sqrt{V_{i-1}^{2}+2 \cdot g^{\prime} L_{i}\left(i_{i}-w_{0(i)}-w_{\mathrm{sn}(i)}-w_{\mathrm{b}(i)}\right) \cdot 10^{-3}}
$$

where $L_{i}, i_{i}$ - the length and slope of $i$ th technological element, respectively. Since the $H_{\mathrm{YRP}}$ is defined under favourable rolling conditions, $w_{\mathrm{sn}(i)}$ and, besides, according to [1] $w_{\mathrm{b}(i)}=0$.

Thus,

$$
V_{i}^{\prime}=\sqrt{V_{i-1}^{2}+2 \cdot g^{\prime} L_{i}\left(i_{i}-w_{0(i)}\right) \cdot 10^{-3}}
$$

where $V_{i-1}$ - the heavy car speed at the end of $i-1$ element in a second approximation (with consideration of $w_{\mathrm{rol}(i-1)}$ and $\left.w_{\mathrm{b}(i-1)}\right)$.

The heavy car speed at the end of $m$ th element:

$$
\begin{aligned}
V_{m}= & \left(V_{0}^{2}+2 \cdot g^{\prime} L_{1}\left(i_{1}-w_{0}-w_{\mathrm{rol}(1)}-w_{\mathrm{b}(1)}\right) \cdot 10^{-3}\right. \\
& \left.+2 \cdot g^{\prime} L_{2}\left(i_{2}-w_{0}-w_{\mathrm{rol}(2)}-w_{\mathrm{b}(2)}\right) \cdot 10^{-3}+\cdots+2 \cdot g^{\prime} L_{m}\left(i_{m}-w_{0}-w_{\mathrm{rol}(m)}-w_{\mathrm{b}(m)}\right) \cdot 10^{-3}\right)^{1 / 2} \\
& =\sqrt{V_{0}^{2}+2 \cdot g^{\prime} \sum_{s=1}^{m} L_{s}\left(i_{s}-w_{0}-w_{\mathrm{rol}(s)}-w_{\mathrm{b}(s)}\right) \cdot 10^{-3}}
\end{aligned}
$$

Therefore, the heavy car speed at the origin of $(i-1)$ th element is:

$$
V_{i-1}=\sqrt{V_{0}^{2}+2 \cdot g^{\prime} \sum_{s=1}^{i-1} L_{s}\left(i_{s}-w_{0}-w_{\mathrm{rol}(s)}-w_{\mathrm{b}(s)}\right) \cdot 10^{-3}} .
$$


According to [1], the actual average rolling speed of a heavy car on the technological element is:

$$
V_{\mathrm{mid}}^{a}=\frac{V_{\mathrm{st}}+\sum_{i=1}^{Z} \sqrt{V_{\mathrm{st}}^{2}+2 g^{\prime} L_{i}\left(i_{i}-w_{0}\right) /(1000 Z)}}{Z+1},
$$

where $Z$ - the number of elemental sections as components of the technological element (for calculation of $V_{\text {mid }}^{a}$, the elemental section length is taken $0.5 \mathrm{~m}$, i.e. $L / Z=0.5$ ). According to [1], the authors propose to define the average speed on a technological element by the formula:

$$
V_{\operatorname{mid}(i)}=k_{i} V_{\mathrm{m}(i)}^{\prime},
$$

where $V_{\mathrm{m}(i)}^{\prime}$ - the heavy car's speed in a first approximation in the middle of a technological element:

$$
V_{\mathrm{m}(i)}=\sqrt{V_{i-1}^{2}+g^{\prime} L_{i}\left(i_{i}-w_{0}^{\mathrm{VRG}}\right) \cdot 10^{-3}}
$$

and $k_{i}$ - the correction index.

The authors propose to define the correction index on the basis of the conditions of equality of errors in calculation of the average heavy car speed on technological elements; one of them is of an infinitesimal length, thus, $V_{\text {mid }}$ on the element can be taken as $V_{\text {st }}$, the second - as a length of $30 \mathrm{~m}$ and located on a slope of $50 \% 0$, thus:

$$
V_{\mathrm{st}}-k V_{\mathrm{st}}^{\prime}=k V_{m}^{\prime}-V_{\mathrm{mid}}^{a} .
$$

From the equation obtained:

$$
k=\frac{V_{\mathrm{st}}+V_{\mathrm{mid}}^{a}}{V_{\mathrm{st}}+V_{m}^{\prime}} .
$$

The research made by the authors on the basis of calculations of the correction index at various $V_{\text {st }}$ showed that it can be set as an exponential function:

$$
k=-0.0576191 \cdot e^{-0.5710201 \cdot V_{\mathrm{st}}}+0.9966873 .
$$

Thus,

$$
H_{\mathrm{YRP}}=A\left(\sum_{i=1}^{m}\left(L_{i} i_{i}\right) \cdot 10^{-3}+B+\sum_{i-1}^{m} C_{i} k_{i}^{2} V_{i-1}^{2} g^{\prime} L_{i}\left(i_{i}-w_{0}^{\mathrm{VGC}}\right) \cdot 10^{-3}\right) \rightarrow H_{\mathrm{YRP}(\min )}
$$

where

$$
\begin{aligned}
V_{i-1}^{2}=V_{0}^{2}+2 g_{\mathrm{VGC}}^{\prime} & \sum_{s=1}^{i-1}\left(L_{s}\left(i_{s}-w_{0}^{\mathrm{VGC}}\right) \cdot 10^{-3}\right. \\
& -\left(0.56 \cdot n_{\mathrm{switch}(s)}+0.23 \sum a_{s}\right) k_{s}^{2}\left(V_{s-1}^{2}+g^{\prime} L_{g}\left(i_{g}-w_{0}^{\mathrm{VGC}}\right) \cdot 10^{-3}-1000 \cdot h_{b}(s)\right) .
\end{aligned}
$$

Let:

$$
C_{i} k_{i}^{2}=D_{i}, \quad V_{i-1}^{2}-g^{\prime} L_{i} w_{o} \cdot 10^{-3}=E_{i}, \quad g^{\prime} L_{i} \cdot 10^{-3}=F_{i}, \quad L_{i} \cdot 10^{-3}=G_{i} .
$$

Then:

$$
H_{\mathrm{YRP}}=A\left(\sum_{i-1}^{m} G_{i} i_{i}+B+\sum_{i=1}^{m} D_{i}\left(E_{i}+F_{i} i_{i}\right)\right) \rightarrow H_{\mathrm{YRP}(\min )}
$$

or

$$
\begin{aligned}
H_{\mathrm{YRP}}=A\left(B+\left(\left(G_{1} i_{1}\right)+D_{1}\left(E_{1}+F_{1} i_{1}\right)\right)+\left(\left(G_{2} i_{2}\right)\right.\right. & \left.+D_{2}\left(E_{2}+F_{2} i_{2}\right)\right) \\
& \left.+\cdots+\left(\left(G_{m} i_{m}\right)+D_{m}\left(E_{m}+F_{m} i_{m}\right)\right)\right) \rightarrow H_{\mathrm{YRP}(\min )} .
\end{aligned}
$$

As far as each profile element consists of the several technological elements, let us specify:

$$
\begin{aligned}
& I_{1}=i_{i}, \quad \text { where } i=1, \ldots, X_{1}, \\
& I_{2}=i_{i}, \quad \text { where } i=X+1, \ldots, X_{2}, \\
& I_{n}=i_{i}, \quad \text { where } i=X_{n-1}+1, \ldots, X_{n} .
\end{aligned}
$$

where $n$ - the number of profile elements of the descent part of a hump, and $X_{i}, i=1,2, \ldots, n$ - the number of the last technological element of the $i$ th profile element 
And, eventually, the objective function takes the form:

$$
\begin{aligned}
H_{\mathrm{YRP}}=A\left(B+\sum_{i=1}^{X_{1}}\left(G_{i} I_{1}+D_{i}\left(E_{i}+F_{i} I_{1}\right)\right)\right. & +\sum_{i=X_{1}+1}^{X_{2}}\left(G_{i} I_{2}+D_{i}\left(E_{i}+F_{i} I_{2}\right)\right) \\
& \left.+\cdots+\sum_{i=X_{n-1}+1}^{X_{n}}\left(G_{i} I_{n}+D_{i}\left(E_{i}+F_{i} I_{n}\right)\right)\right) \rightarrow H_{\mathrm{YRP}(\min )}
\end{aligned}
$$

In order to realize the guided gravity technology for cuts, the objective function (23) should be minimized at non-linear limitations-equalities:

$$
\left\{\begin{array}{l}
D_{1}=f_{D_{1}}\left(V_{0}\right), E_{1}=f_{E_{1}}\left(V_{0}\right), \\
D_{2}=f_{D_{2}}\left(V_{0}, i_{1}\right), E_{2}=f_{E_{2}}\left(V_{0}, i_{1}\right), \\
D_{3}=f_{D_{3}}\left(V_{0}, i_{1}, i_{2}\right), E_{3}=f_{E_{3}}\left(V_{0}, i_{1}, i_{2}\right), \\
D_{X_{n}}=f_{D_{X_{n}}}\left(V_{0}, i_{1}, i_{2}, \ldots, i_{X_{n-1}}\right), E_{n}=f_{E_{X_{n}}}\left(V_{0}, i_{1}, i_{2}, \ldots, i_{X_{n-1}}\right),
\end{array},\right.
$$

linear limitations-inequalities:

$$
\left\{\begin{array}{l}
0 \leq I_{1} \leq 50 \\
25 \leq I_{2} \leq 50 \\
-50 \leq I_{n} \leq 50 \\
I_{1}-I_{2} \leq 25 \\
H_{b}^{\mathrm{YRP}} \leq n_{r} h_{\mathrm{one}} \\
V_{e n}^{\mathrm{YRP}} \leq V_{\mathrm{en}(\max )}^{\mathrm{mR}} \\
T_{0} \leq T_{0}^{\max }
\end{array}\right.
$$

and linear limitations-equalities:

$$
\left\{\begin{array}{l}
L_{\text {run }}^{\text {unf }}=L_{c}, \\
V_{e x}^{\mathrm{YHR}}=1.4,
\end{array}\right.
$$

where $I_{1}, I_{2}, \ldots, I_{n}$ - the profile element slopes of a hump, $\%_{0} ; H_{B}^{\mathrm{YRP}}$ - the value of the heavy car braking under favourable summer conditions at YRP, $k J / k N ; n_{r}$ - the number of retarders installed at YRPs; $h_{\mathrm{one}}-$ the capacity of a retarder installed at YRP, $k J / k N ; V_{e n}^{\mathrm{YRP}}$ - the entry speed of a heavy car under favourable summer conditions at $\mathrm{YRP}, \mathrm{m} / \mathrm{s} ; V_{\mathrm{en}(\max )}^{\mathrm{YRP}}$ - the maximum admissible entry speed of a heavy car under favourable summer conditions at YRP, $m / s ; T_{0}$ - the time interval at dividing elements between cars rolling in turn, sec; $T_{0}^{\max }$ - the maximum accessible time at dividing elements between cars rolling in turn, sec; $L_{\text {run }}^{\text {unf }}-$ the run of a heavy car under unfavourable winter conditions along a difficult track in terms of resistance, $m$; $L_{c}-$ the calculation length of a difficult track in terms of resistance from the hump crest to the design point, $m$; $V_{e x}^{\mathrm{YRP}}-$ the exit speed of a heavy car from YRP, $m / s$. According to [8], the task cannot be reduced to an unconditional extremum task.

Therefore, let us consider other ways to solve the task.

The method of Lagrange multipliers is technically difficult to implement as the number of limitations-equalities is rather great. However, the method cannot be directly used if limitations are inequalities [8].

Thus, there is a needed to have a method that will allow finding the minimal value of $H_{\mathrm{YRP}}$ with the minimal variant search.

The standard method of Lagrange multipliers supplemented with terms, stem from the duality theory, got its generalization to the task of non-linear programming of a general kind with limitations of the equality-inequality type 8 .

The needed optimality conditions of such tasks are called the Kuhn-Tucker conditions. In order to build the Kuhn-Tucker tasks, a mathematical model of the non-linear programming task must have a strict limitationsinequalities recording:

$$
\begin{aligned}
& Z=f(X) \rightarrow \min \\
& \left\{\begin{array}{l}
h_{k}(X)=0, k=\overline{1, s} \\
g_{i}(X) \leq 0, i=\overline{1, m}
\end{array}\right.
\end{aligned}
$$

The conditions of non-negative variables are included in the task recording as limitations-inequalities:

$$
g_{i}=-x_{j} \leq 0
$$


The Lagrange function of the task is built with $s+m$ of undetermined coefficients:

$$
L(X, V, U)=f(X)+\sum_{k=1}^{s} V_{k} h_{k}(X)+\sum_{i=1}^{m} U_{i} g_{i}(X) .
$$

The coefficients $V_{k}(k=\overline{1, s}), U_{i}(i=\overline{1, m})$ are called the Lagrange multipliers. They are unlimited by sign dual variables corresponding to limitations-equalities $\left(V_{k}=0, k=\overline{1, s}\right)$ and non-negative dual variables corresponding to limitations-inequalities $\left(U_{i} \geq 0, i=\overline{1, m}\right)$.

The following equation system with the $n+s+m$ unknown variable is called the Kuhn-Tucker task for minimization:

$$
\left\{\begin{array}{l}
\vec{\nabla} f(X)+\sum_{k=1}^{s} V_{k} \vec{\nabla} h_{k}(X)+\sum_{i=1}^{m} U_{i} \vec{\nabla} g_{i}(X)=0 \\
h_{k}(X)=0, k=\overline{1, s} \\
g_{i}(X) \leq 0, i=\overline{1, m} \\
U_{i} g_{i}(X)=0, i=\overline{1, m} \\
U_{i} \geq 0, i=\overline{1, m}
\end{array}\right.
$$

The equations $U_{i} g_{i}(X)=0, i=\overline{1, m}$ are the complementary slackness conditions; they are an analogy of the second duality theorem of linear programming tasks. If in point $X$, the limitation $g_{i}(X)$ is inactive $\left(g_{i}(X)>0\right)$ then $U_{i}=0$, if $g_{i}(X)$ is active $\left(g_{i}(X)=0\right)$, than $U_{i}>0$.

The solution to the Kuhn-Tucker task should start with the analysis of this group of equations searching all possible combinations of equality to zero $U_{i}$ or $g_{i}(X)$ in turn. The optimal solution should be sought among points meeting the Kuhn-Tucker conditions (30). Let us build the Kuhn-Tucker task at linear limitations-equalities for output task 23 :

$$
\left\{\begin{array}{l}
L_{\mathrm{run}}^{\mathrm{unf}}-L_{c}=0, \\
V_{e x}^{\mathrm{PHR}}-1.4=0,
\end{array}\right.
$$

non-linear limitations-equalities:

$$
\left\{\begin{array}{l}
D_{1}-f_{D_{1}}\left(V_{0}\right)=0 \\
E_{1}-f_{E_{1}}\left(V_{0}\right)=0, \\
D_{2}-f_{D_{2}}\left(V_{0}, I_{1}\right)=0 \\
E_{2}-f_{E_{2}}\left(V_{0}, I_{1}\right)=0 \\
D_{3}-f_{D_{3}}\left(V_{0}, I_{1}, I_{2}\right)=0 \\
E_{3}-f_{E_{3}}\left(V_{0}, I_{1}, I_{2}\right)=0 \\
\vdots \\
D_{Z_{x}}-f_{D_{Z_{x}}}\left(V_{0}, I_{1}, I_{2}, \ldots, I_{Z_{x-1}}\right)=0 \\
E_{Z_{x}}-f_{E_{Z_{x}}}\left(V_{0}, I_{1}, I_{2}, \ldots, I_{Z_{x-1}}\right)=0
\end{array}\right.
$$

linear limitations-inequalities:

$$
\begin{gathered}
0 \leq I_{1} \leq 50 \rightarrow\left\{\begin{array}{l}
0-I_{1} \leq 0, \\
I_{1}-50 \leq 0,
\end{array}\right. \\
25 \leq I_{2} \leq 50 \rightarrow\left\{\begin{array}{l}
25-I_{2} \leq 0, \\
I_{2}-50 \leq 0,
\end{array}\right. \\
\vdots \\
-50 \leq I_{n} \leq 50 \rightarrow\left\{\begin{array}{l}
-50-I_{n} \leq 0, \\
I_{n}-50 \leq 0 ;
\end{array}\right.
\end{gathered}
$$

and

$$
\left\{\begin{array}{l}
I_{1}-I_{2}-25 \leq 0 \\
V_{e n}^{\mathrm{YRP}}-V_{\mathrm{en}(\max )}^{\mathrm{YRP}} \leq 0 \\
T_{0}-T_{0}^{\max } \leq 0
\end{array}\right.
$$


The Lagrange function has the form:

$$
\begin{gathered}
L(I, V, U)=A\left(B+\sum_{j=1}^{Z_{1}}\left(C_{j} I_{1}+D_{j}\left(E_{j}+F_{j} I_{1}\right)\right)+\sum_{j=Z_{1}+1}^{Z_{2}}\left(C_{j} I_{2}+D_{j}\left(E_{j}+F_{j} I_{2}\right)\right)\right. \\
\left.+\cdots+\sum_{j=Z_{x-1}+1}^{Z_{x}}\left(C_{j} I_{x}+D_{j}\left(E_{j}+F_{j} I_{x}\right)\right)\right)+V_{1}\left(L_{\mathrm{run}}^{\mathrm{unf}}-L_{c}\right)+V_{2}\left(V_{e x}^{\mathrm{YRP}}-1.4\right)+V_{3}\left(D_{1}-f_{D_{1}}\left(V_{0}\right)\right) \\
+V_{4}\left(E_{1}-f_{E_{1}}\left(V_{0}\right)\right)+V_{5}\left(D_{2}-f_{D_{2}}\left(V_{0}, I_{1}\right)\right)+V_{6}\left(E_{2}-f_{E_{2}}\left(V_{0}, I_{1}\right)\right)+V_{7}\left(D_{3}-f_{D_{3}}\left(V_{0}, I_{1}, I_{2}\right)\right)+V_{8}\left(E_{3}-f_{E_{3}}\left(V_{0}, I_{1}, I_{2}\right)\right) \\
\quad+\cdots+V_{2 Z_{x+2}}\left(D_{Z_{x}}-f_{D_{Z_{x}}}\left(V_{0}, I_{1}, I_{2}, \ldots I_{Z_{x-1}}\right)\right)+V_{2 Z_{x+3}}\left(E_{Z_{x}}-f_{E_{Z_{x}}}\left(V_{0}, I_{1}, I_{2}, \ldots I_{Z_{x-1}}\right)\right) \\
+U_{1}\left(0-I_{1}\right)+U_{2}\left(I_{1}-50\right)+U_{3}\left(25-I_{2}\right)+U_{4}\left(I_{2}-50\right)+\cdots+U_{2 x-1}\left(-50-I_{x}\right)+U_{2 x}\left(I_{x}-50\right) \\
\quad+U_{2 x+1}\left(I_{1}-I_{2}-25\right)+U_{2 x+2}\left(V_{e n}^{\mathrm{YRP}}-V_{\mathrm{en}(\max )}^{\mathrm{YRP}}\right)+U_{2 x+3}\left(T_{0}-T_{0}^{\mathrm{max}}\right), \quad(35)
\end{gathered}
$$

and conditions:

(1.)

$$
\begin{gathered}
A\left(\sum_{j=1}^{Z_{1}}\left(G_{j}+D_{j} F_{j}\right)\right)-V_{6} \frac{\partial f_{D_{2}}}{\partial I_{1}}-V_{7} \frac{\partial f_{E_{2}}}{\partial I_{1}}-V_{8} \frac{\partial f_{D_{3}}}{\partial I_{1}}-V_{9} \frac{\partial f_{E_{3}}}{\partial I_{1}} \\
-\cdots-V_{2 Z_{x}+2} \frac{\partial f_{D_{Z_{x}}}}{\partial I_{1}}-V_{2 Z_{x}+3} \frac{\partial f_{E_{Z_{x}}}}{\partial I_{1}}-U_{1}+U_{2}+U_{2 x+1}=0 \\
\vdots \\
A\left(\sum_{j=Z_{x-1}+1}^{Z_{x}}\left(G_{j}+D_{j} F_{j}\right)\right)-U_{2 x-1}-U_{2 x}=0
\end{gathered}
$$

(2.) partial derivatives by dual variables $\frac{\partial L}{\partial V_{k}}$ and $\frac{\partial L}{\partial U_{i}}$ :

$$
\begin{gathered}
L_{\mathrm{run}}^{\mathrm{unf}}-L_{c}=0 \\
V_{e x}^{\mathrm{YRP}}-1.4=0, \\
D_{1}-F_{D_{1}}\left(V_{0}\right)=0, E_{1}-f_{E_{1}}\left(V_{0}\right)=0, \\
D_{2}-F_{D_{2}}\left(V_{0}, I_{1}\right)=0, E_{2}-f_{D_{2}}\left(V_{0}, I_{1}\right)=0, \\
D_{3}-F_{D_{3}}\left(V_{0}, I_{1}, I_{2}\right)=0, E_{3}-f_{E_{3}}\left(V_{0}, I_{1}, I_{2}\right)=0, \\
\vdots \\
D_{Z_{x}}-f_{D_{Z_{x}}}\left(V_{0}, I_{1}, I_{2}, \ldots, I_{Z_{x-1}}\right)=0, \\
E_{Z_{x}}-f_{E_{Z_{x}}}\left(V_{0}, I_{1}, I_{2}, \ldots, I_{Z_{x-1}}\right)=0, \\
0-I_{1}=0 ; I_{1}-50=0 \\
25-I_{2}=0, I_{2}-50=0 \\
\vdots \\
-50-I_{x}=0 ; I_{x}-50=0 ; I_{1}-I_{2}-25=0, \\
V_{e n}^{\mathrm{YRP}}-V_{\mathrm{en}(\max )}^{\mathrm{YRP}}=0 ; T_{0}-T_{0}^{\max }=0 ;
\end{gathered}
$$

(3.) the complementary slackness condition (2nd duality theorem):

$$
\begin{gathered}
U_{1}\left(0-I_{1}\right)=0, \\
U_{2}\left(I_{1}-50\right)=0, \\
U_{3}\left(25-I_{2}\right)=0, \\
U_{4}\left(I_{2}-50\right)=0, \\
\vdots \\
U_{2 x-1}\left(-50-I_{X}\right)-0=0, \\
U_{2 x}\left(I_{x}-50\right)=0, \\
U_{2 x+1}\left(I_{1}-I_{2}-25\right)=0,
\end{gathered}
$$




$$
\begin{gathered}
U_{2 x+2}\left(V_{e n}^{\mathrm{YRP}}-V_{\mathrm{en}(\max )}^{\mathrm{YRP}}\right)=0, \\
U_{2 x+3}\left(T_{0}-T_{0}^{\max }\right)=0
\end{gathered}
$$

(4.)

$$
U_{i} \geq 0
$$

According to [8], the solution should start with a linear search of all possible combinations of equality to zero for multipliers of group III. At first, let us suppose that dual variables are equal to zero or not equal to zero: $U_{i}=0(\forall i)$ then one out of $U_{i}=0$, the others are not equal to zero, etc.

\section{Conclusions}

The solution to the optimization task with limitations presented in the study will make it possible to minimize the demand for the capacity of braking facilities and implement the technology of guided gravity regulation of the cut speed. The authors believe that the implementation of the technology will encourage the decrease of operational costs for a refund of a car and freight damages (due to better conditions improving the quality of the cut rolling speed regulation), and for electricity needed for this regulation (a possible decrease of wind consumption by car retarders). Besides, it will reduce additional charges for demurrages caused by waiting for breaking-up at arrival yards (due to a possible reduction of hump intervals and lower volume of marshalling works for pulling cars with a subsequent remedy of the consequences).

\section{REFERENCES}

[1] M. I. Kutsenko, I. V. Berestov, O. M. Ohar, O. B. Akhiiezer. As for the question of development of methodology of complex calculation of optimal structural parameters of gravity hump. Eastern-European Journal of Enterprise Technologies (2/3):56-60, 2009.

[2] V. I. Bobrovskiy, A. Kolesnik, A. S. Dorosh. The perfection of construction of plan of the track development of humping access door. Transport systems and transportations technologies : collection of research papers Dnipropetrovsk national University of railway transport named by academic V Lazaryan (1):27-33, 2001.

[3] E. A. Mamaev. The perfection of construction of plan of the track development of humping access door, vol.11. International Journal of Applied Engineering Research (23):11515-11524, 2016.

[4] S. A. Bessonenko. The optimization of basic parameters of gravity humps. The perfection of railways operating work, Novosibirsk (1):4-25, 2008.

[5] V. A. Kobzev. The state and prospects of braking hill technique development. Automation, connection, informatics (11):2-5, 2004

[6] A. N. Shabelnikov. The development of theory and methods of automation of management with difficult processes at the marshalling yard. First printing. Russian State technical university, Moscow, 2005.

[7] V. I. Bobrovskiy, D. N. Kozachenko, N. P. Bozhko, et al. The optimization of the modes of uncoupling braking on gravity humps. First printing. Makovets, Dnepropetrovsk, 2010.

[8] T. A. Hemdi. Introduction to the analysis of operations. First printing. Vilyams, St-Peterburg, 2007. 